\title{
Graphene materials with different structures prepared from the same graphite by the Hummers and Brodie methods
}

\author{
Cristina Botas ${ }^{a}$, Patricia Álvarez ${ }^{a}$, Patricia Blanco ${ }^{a}$, Marcos Granda $^{a}$, Clara Blanco ${ }^{a}$, Ricardo \\ Santamaría $^{a}$, Laura J. Romasanta ${ }^{b}$, Raquel Verdejo ${ }^{b}$, Miguel A. López-Manchado ${ }^{b}$ and Rosa \\ Menéndez ${ }^{a, *}$ \\ ${ }^{a}$ Instituto Nacional del Carbón, INCAR-CSIC, Apartado 73, 33080 Oviedo, Spain.

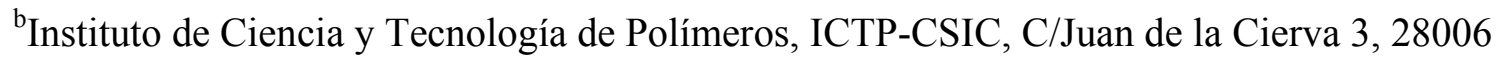 \\ Madrid, Spain.
}

ABSTRACT. Graphene materials containing different functional groups were prepared from a natural graphite, by means of two different oxidation methods (Hummers and Brodie). It was observed that the differences in the structure of the resultant graphite oxides (GOs) greatly affect the structure of the graphenes resulting from their thermal exfoliation/reduction. Although the oxidation of the graphite was more effective with the modified Hummers method than with Brodie's method ( $\mathrm{C} / \mathrm{O}$ of 1.8 vs 2.9 , as determined by XPS), the former generated a lower residual oxygen content after thermal exfoliation/reduction and a better reconstruction of the 2D graphene structure (with fewer defects). This is explained by the presence of conjugated epoxy and hydroxyl groups in the GO obtained by Brodie's method, which upon thermal treatment, lead to the incorporation of oxygen into the carbon lattice preventing its complete restoration. Additionally, graphene materials obtained with Brodie's method exhibit, in general, a smaller sheet size and larger surface area.

\footnotetext{
* Corresponding author: Tel: +34 9851190 90. E-mail: rosmenen@incar.csic.es (Rosa Menéndez)
} 


\section{INTRODUCTION}

The development of graphene materials of different structure, functionality and sheet size is of special interest for different applications as for example polymer composites [1,2]. The presence of polar groups on graphene surface can improve the compatibility with the polymer matrices but reduces its inherent thermal and electrical conductivity $[3,4,5]$. The chemical route through the oxidation of graphite is widely used due to both the easy scalability of the process and the costs involved [1, 2]. Furthermore, the possibility of using different parent graphites [6], oxidation methods [7] (or conditions) [8] and different reduction processes widens the range of graphene materials that can be produced $[7,9,10]$. Although the structure of graphene oxides $(\mathrm{GrO})$ is still a matter of debate the last studies seem to confirm that they exhibit epoxy, hydroxyl (mainly at the basal planes of the sheets) and carboxyl groups (at the edges of the sheets or the defects (pores) $[11,12,13]$. Nonetheless, the amount type and location of the oxygen functional groups can be varied by modifying the preparation conditions, which could have a strong influence on the reactivity of these materials. For instance, depending on the quantity and location of theses groups, the GrO will exhibit a very different behavior, either when used directly for a specific application (catalysis) or when it is subjected to further reduction treatment [9, 14]. Carboxyl groups and hydroxyl and epoxy groups located on the basal plane (in the interior of the sheet) are the most reactive on thermal reduction. Hydroxyl and epoxy groups located on the edges exhibit a lower reactivity [15]. In addition, the relative contribution of one or another group (i.e. hydroxyl/epoxy) and their proximity to each other need to be considered [15]. The yield and size of the sheets of the graphene materials can also be controlled by varying the crystal structure of the parent graphite [6] and/or the graphite oxide exfoliation conditions [16]. 
Thermal reduction has many advantages over chemical reduction for the restoration of the pristine graphite 2D-structure through the elimination of oxygen functional groups. It is simpler and easier to perform, as it usually results in the simultaneous exfoliation and reduction of the graphite oxide. Furthermore, there is no need to use liquids [16], which is an advantage for some applications, for example as electrodes in lithium [17] or vanadium batteries [18], which require dry graphene. Moreover, when large amounts of sample are demanded and the graphene materials need to have a good thermal conductivity, as in the preparation of polymer-based composites with good heat dissipation properties [19], the thermal exfoliation/reduction of graphite oxide is an excellent alternative [18]. The possibility of retaining some oxygen functional groups in the graphene to facilitate interaction with the polymer is an additional advantage.

Nowadays, the most widely used methods to prepare graphite oxide (GO) and/or graphene oxide (GrO) is either the Hummers [20] or the Brodie [21] method. These methods differ in both the acid medium (nitric or sulfuric acid), and the type of salt used (potassium chlorate or potassium permanganate). The oxidation degree attained is usually reported to be higher for the Hummers method. A recent publication evaluated the structural transformations of GO prepared by both methods using solvation/hydration [22]. The solvation/hydration behavior of the GOs obtained by the Brodie (GO-B) and Hummers (GO-H) methods resulted in crystalline and osmotic swelling, respectively. These were ascribed to the presence $\mathrm{C}-\mathrm{OH}$ groups in $\mathrm{GO}-\mathrm{B}$ and $\mathrm{C}=\mathrm{O}$ groups in GO-H, respectively. They also analyzed the thermal exfoliation behavior of both products, and observed a smaller degree of expansion in GO-H compared to GO-B. However to 
our knowledge there have been no studies on the effect of the two methods on the reconstruction of the $\mathrm{sp}^{2} \mathrm{C}$ structure, when thermal treatment is applied to their respective graphene oxides, which is directly related with certain properties of the resultant materials such as their thermal and electrical conductivity.

The overall aim of this research is to obtain polymer-based graphene composites with improved heat transmission properties. For that, in an initial stage, graphene materials with different sized sheets and containing different functional groups were prepared. This paper reports on: i) the oxidation of a natural graphite by the Hummers [20] and Brodie [21] methods; ii) the thermal exfoliation/reduction of the graphite oxides (GOs) at temperatures of 700,1000 and $2000{ }^{\circ} \mathrm{C}$ [23]; iii) the characterization of the graphene materials obtained by the two methods.

\section{EXPERIMENTAL SECTION}

A commercial natural graphite powder supplied by Sigma Aldrich was used as starting material for the preparation of the samples in this study. The ash content of the graphite, as determined by TGA was lower than $0.1 \mathrm{wt} \%$. The carbon content, on an ash-free basis was $99.9 \mathrm{wt} \%$. The characterization data of the graphite are included in the Supporting Information (S.I.).

\subsection{Preparation of graphite oxide by a modified version Hummers method}

GO was prepared from the commercial graphite using a modified Hummers' method (GO-H) [6, 20, 23]. This method makes use of the Hummers' reagents with additional amounts of $\mathrm{NaNO}_{3}$ and $\mathrm{KMnO}_{4}$. Concentrated $\mathrm{H}_{2} \mathrm{SO}_{4}(360 \mathrm{~mL})$ was added to a mixture of graphite $(7.5 \mathrm{~g})$ and $\mathrm{NaNO}_{3}(7.5 \mathrm{~g})$, and the mixture was cooled down to $0{ }^{\circ} \mathrm{C}$ in an ice bath. $\mathrm{KMnO}_{4}(45 \mathrm{~g})$ was 
added slowly in small doses to keep the reaction temperature below $20{ }^{\circ} \mathrm{C}$. The solution was heated to $35{ }^{\circ} \mathrm{C}$ and stirred for $3 \mathrm{~h}$. Then $3 \% \mathrm{H}_{2} \mathrm{O}_{2}(1.5 \mathrm{~L})$ was slowly added. This had a pronounced exothermal effect at $98{ }^{\circ} \mathrm{C}$. The reaction mixture was stirred for $30 \mathrm{~min}$ and, finally, the mixture was centrifuged (3700 rpm for $30 \mathrm{~min}$ ), after which the supernatant was decanted away. The remaining solid material was then washed with $600 \mathrm{~mL}$ of water and centrifuged again, this process being repeated until the $\mathrm{pH}$ was neutral [6].

\subsection{Preparation of graphite oxide by the Brodie method}

The oxidation of the graphite was also performed using Brodie's method (GO-B) [21]. Fuming nitric acid $(200 \mathrm{~mL})$ was added into a flask with a cooling jacket and cooled to $0{ }^{\circ} \mathrm{C}$ in a cryostat bath. The graphite powder ( $10 \mathrm{~g})$ was introduced into the flask and thoroughly dispersed to avoid agglomeration. Next, potassium chlorate $(80 \mathrm{~g})$ was slowly added for $1 \mathrm{~h}$, and the reaction mixture was stirred for $21 \mathrm{~h}$ at $0{ }^{\circ} \mathrm{C}$. Special caution is necessary during addition of potassium chlorate since explosions can occur [3]. Once the reaction had finished, the mixture was diluted in distilled water and vacuum filtered until the $\mathrm{pH}$ of the filtrate was neutral.

\subsection{Thermal exfoliation/reduction of GOs}

The temperatures used for the exfoliation/reduction of GO-H and GO-B to prepare the graphene materials (TRGs) were 700,1000 and $2000{ }^{\circ} \mathrm{C}$. The treatments at 700 and $1000{ }^{\circ} \mathrm{C}$ were performed in a horizontal tube furnace using a ceramic boat with a graphite cover to prevent the blowing of the material [23]. $0.3 \mathrm{~g}$ of GO was introduced into the furnace and heated at $5{ }^{\circ} \mathrm{C} \mathrm{min}-$

${ }^{1}$ under a $\mathrm{N}_{2}$ atmosphere $\left(100 \mathrm{~mL} \mathrm{~min}^{-1}\right)$ to the desired temperature, and kept for $1 \mathrm{~h}$. The samples obtained at $700{ }^{\circ} \mathrm{C}$ were then annealed at $2000{ }^{\circ} \mathrm{C}$ in a graphitization furnace (Pyrox VI 
$150 / 125)$ under an atmosphere of $\operatorname{argon}\left(3 \mathrm{~L} \mathrm{~min}^{-1}\right)$ at a heating rate of $5{ }^{\circ} \mathrm{C} \min ^{-1}$ up to $800{ }^{\circ} \mathrm{C}$ and then at $10{ }^{\circ} \mathrm{C} \min ^{-1}$ up to $2000{ }^{\circ} \mathrm{C}$, this temperature being maintained for $1 \mathrm{~h}$. The samples obtained were labeled as TRGH-700, TRGH-1000, TRGH-2000, TRGB-700, TRGB-1000 and TRGB-2000, where $\mathrm{H}$ and $\mathrm{B}$ refer to the oxidation method ( $\mathrm{H}$ : Hummers and B: Brodie). Colloidal suspensions of individual TRG sheets were prepared in purified water/DMF (1:1) in 1 $\mathrm{mL}$ batches and kept under ultrasound treatment for $30 \mathrm{~min}$.

\subsection{Characterization of the samples}

The GOs were thermally treated in a thermal programmed desorption (TPD) device in order to determine the temperature of their thermal exfoliation (blasting temperature) [23]. The system consists of an electrical furnace with a U-shape quartz glass reactor connected to a mass spectrometer (Omnistar TM-Pheiffer Vacuum). Initially, the samples $(50 \mathrm{mg})$ were degassed under a He flow $\left(50 \mathrm{~mL} \mathrm{~min}^{-1}\right)$ at room temperature for $1 \mathrm{~h}$. Then they were heated from room temperature, at a heating rate of $5{ }^{\circ} \mathrm{C} \mathrm{min}^{-1}$, until blasting occurred as a consequence of the sudden release of gases $[16,23]$. Thermogravimetric analyses were carried out using a TA SDT 2960 analyzer. $5 \mathrm{mg}$ of sample was placed in a crucible that was then introduced into the thermobalance; the temperature was increased to $1000{ }^{\circ} \mathrm{C}$ at a heating rate of $5{ }^{\circ} \mathrm{C} \mathrm{min}^{-1}$ under a nitrogen flow of $100 \mathrm{~mL} \mathrm{~min}^{-1}$.

The oxygen content of the samples was determined directly in a LECO-TF-900 furnace coupled to a LECO-CHNS-932 microanalyzer. The analyses were performed using $1 \mathrm{mg}$ of ground sample. The results were quoted from an average of the values of four determinations. In all cases, the experimental error was $<0.5 \%$ of the absolute value. UV-Vis spectra of GrOs were 
recorded at room temperature between 190 and $600 \mathrm{~nm}$ using a UV-Vis spectrometer (UV spectrophotometer. UV-1800, Shimadzu). XPS analyses were carried out in a VG-Microtech Mutilab 3000 device. The XPS C1s peak was analyzed using a peak synthesis procedure that employs a combination of Gaussian and Lorentzian functions [24] in order to identify the functional groups and the respective percentages. The binding energy profiles were deconvoluted as follows: undamaged structures of $\mathrm{Csp}^{2}$-hybridized carbon $(284.5 \mathrm{eV})$, damaged structures or $\mathrm{sp}^{3}$-hybridized carbons (285.5 eV), C-OH groups (286.5 eV), C-O-C functional groups (287.7 $\mathrm{eV})$ and $\mathrm{C}(\mathrm{O}) \mathrm{OH}$ groups at $288.7 \mathrm{eV})$. XRD analysis of the powdered samples was performed using a Bruker D8 Advance diffractometer. The radiation frequency employed was the Ka1 line from $\mathrm{Cu}(1.5406 \AA$ ), with a power supply of $40 \mathrm{kV}$ and $40 \mathrm{~mA}$. The crystallite size along the caxis $\left(L_{c}\right)$ and the interlaminar distances of the sheets were obtained from the (002) reflection of the XRD patterns of the TRGs and the (001) reflection in the case of GOs [23], which were recorded at steps of $0.01^{\circ}$ and intervals of $6 \mathrm{~s}$ per step, using the Scherrer equation. SEM images were obtained using a field emission gun scanning electron microscope (QUANTAN FEG 650, FEI) operating at $5 \mathrm{kV}$. TEM observations were performed on a JEOL 2000 EX-II instrument operating at $160 \mathrm{keV}$. Suspensions of GrOs and TRGs were deposited on standard holey carbon copper grids using the drop cast method and loaded into the microscope. The size and height of the sheets in the GrOs and TRGs suspensions were measured by means of AFM imaging and profiling was carried out by depositing a drop of the suspension onto the surface of mica. The sheets were imaged using a Cervantes atomic force microscope from Nanotec Electronica ${ }^{\mathrm{TM}}$ operating under ambient conditions. Microcantilevers with nominal spring constants of $\mathrm{k}=40$ $\mathrm{N} / \mathrm{m}$ and a resonance frequency of $\mathrm{f}=300 \mathrm{kHz}$ were used to image the sheets. WSxM software was employed to control the atomic force microscope as well as for the data processing of the 
acquired images. Raman spectroscopy was performed on a Renishaw 2000 Confocal Raman Microprobe (Rhenishaw Instruments, England) using a $514.5 \mathrm{~nm}$ argon ion laser. The spectra were recorded from 750 to $3500 \mathrm{~cm}^{-1}$. The surface area was determined from the $\mathrm{N}_{2}$ adsorption isotherm at $77 \mathrm{~K}$ using the BET equation. These analyses were performed in ASAP 2020 Micromeritics equipment using around $100 \mathrm{mg}$ of sample for each experiment. Before the experiments, the samples were outgassed at $350^{\circ} \mathrm{C}$ for $3 \mathrm{~h}$ under vacuum (pressure below $10^{-3}$ $\mathrm{Pa})$.

\section{RESULTS AND DISCUSION}

The parent graphite was fully oxidized by the two methods as confirmed by XRD. The graphite has an intense crystalline peak at $26.5^{\circ}$ corresponding to the (002) plane. On conversion to GO, the (002) and (101) peaks of graphite disappear while the (001) appears at $2 \Theta=9.8^{\circ}$ (Figure 1) [6, 23]. The interlayer distance increases from $0.336 \mathrm{~nm}$ for the graphite to $0.846 \mathrm{~nm}$ for $\mathrm{GO}-\mathrm{H}$ and $0.610 \mathrm{~nm}$ for GO-B, as a result of the expansion caused by the incorporation of water and oxygen functional groups during the oxidation process. The larger value of $\mathrm{GO}-\mathrm{H}$ is the result of a more extensive oxidation as confirmed by elemental analysis. GO-H contains $47.8 \%$ of oxygen, while GO-B only contains $28.2 \%$ (C/O ratios determined by XPS are 1.8 and 2.9, respectively).Also, only in the case of $\mathrm{GO}-\mathrm{H}$ the presence of the small amount of sulphur $(\approx 2$ wt.\%) is observed, as the result of the treatment with sulphuric acid. 


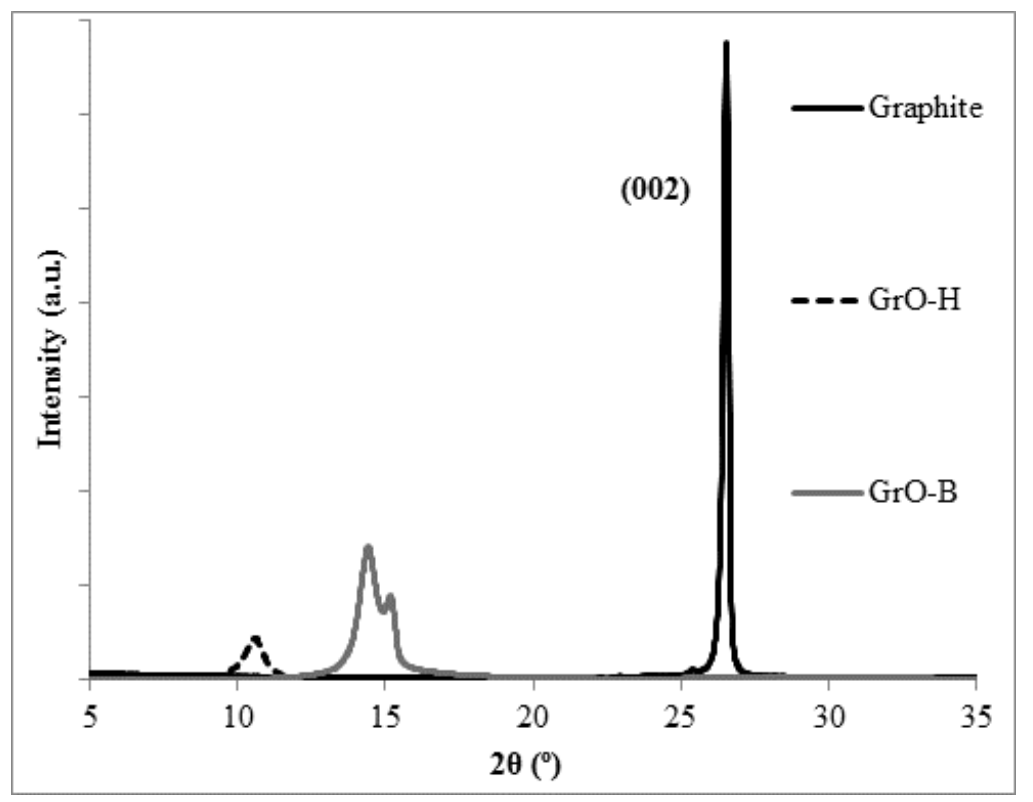

Figure 1. XRD spectra of parent graphite and GOs.

These compositional and structural differences are macroscopically evidenced by the color of their colloidal suspensions in water (Figure 2). GO-H is yellow-brown while GO-B is greenbrown. The facility of GO-H to exfoliate when subjected to ultrasounds (see S.I.), as evidenced by the strong UV-Vis adsorption (Figure 3), is also a consequence of its more extensive oxidation, there being a larger presence of oxygen functional groups which diminishes in a larger extent the Van der Waals interactions $[1,2,6,21]$. Thus, GO-B requires a minimum of $15 \mathrm{~h}$ to exfoliate, whereas GO-H exfoliates after just $1 \mathrm{~h}$. Moreover, the UV-Vis adsorption spectra of the exfoliated GOs are very different. The sample obtained from GO-H exhibits the $230 \mathrm{~nm}$ and $300 \mathrm{~nm}$ peaks typical of graphene oxides, which are attributed to $\pi-\pi^{*}$ transitions of aromatic C$\mathrm{C}$ and $\mathrm{C}-\mathrm{O}$ bonds, respectively (Figure 3), while in the sample from GO-B a multi peak pattern appears above $300 \mathrm{~nm}$ (typical of highly condensed polycyclic aromatic structures). The Raman spectra of both exfoliated samples exhibit clear differences. There is a shift of the G peak position form $1592 \mathrm{~cm}^{-1}$ for GO-H to $1565 \mathrm{~cm}^{-1}$ for GO-B (Table 1) which, according to previous 
studies $[25,26]$ is possibly related to the different distribution of the oxygen functional groups in the graphene sheet..

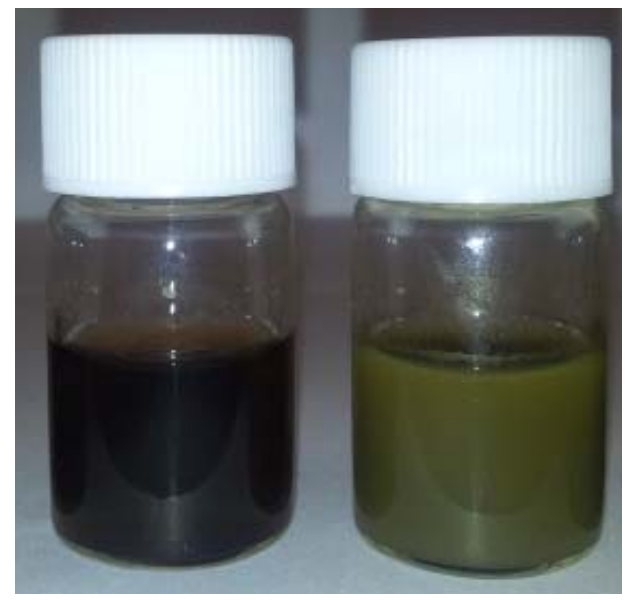

Figure 2. Images of GO-H (left) and GO-B (right) in water (without ultrasonication).

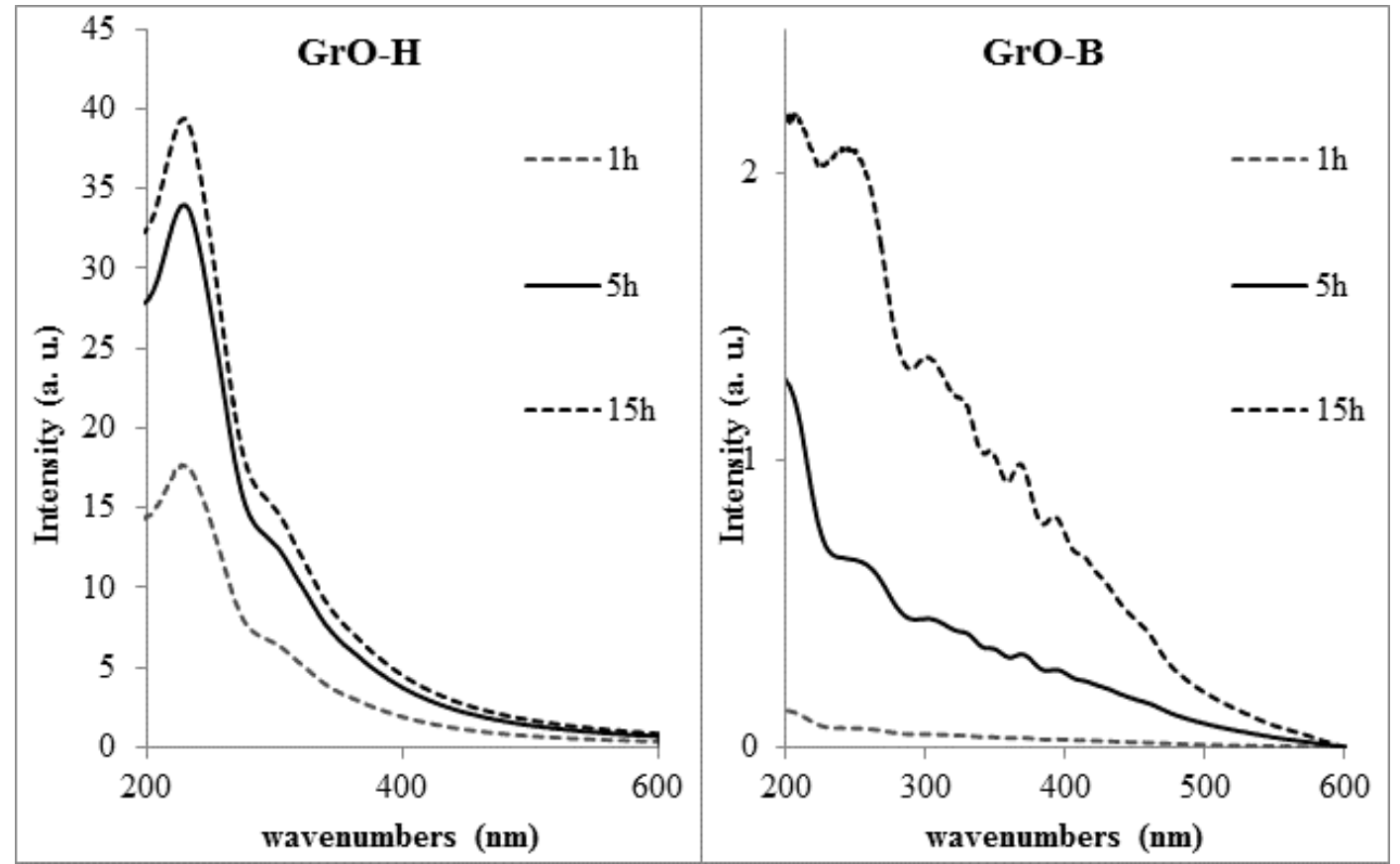


Figure 3. UV-Vis spectra of GrO-H (left) and GrO-B (right) at different sonication times $(1,5$ and $15 \mathrm{~h})$.

The differences in the exfoliation behavior are clearly illustrated in the TEM images which show single folded $\mathrm{GO}-\mathrm{H}$ sheets after dispersion in water and $5 \mathrm{~h}$ sonication (Figure 4) and unexfoliated GO-B for the same sonication time (Figure 4). The larger population of monolayers obtained from GO-H is highlighted by SEM (Figure 5) and AFM (Figure 6). Additionally, GO-H generates larger size sheets than GO-B. This could be due to the poorer degree of exfoliation observed in GO-B (only the smallest particles are exfoliated) or to the breakage of the sheets. In view of what has so far been discussed, the first explanation is more likely.
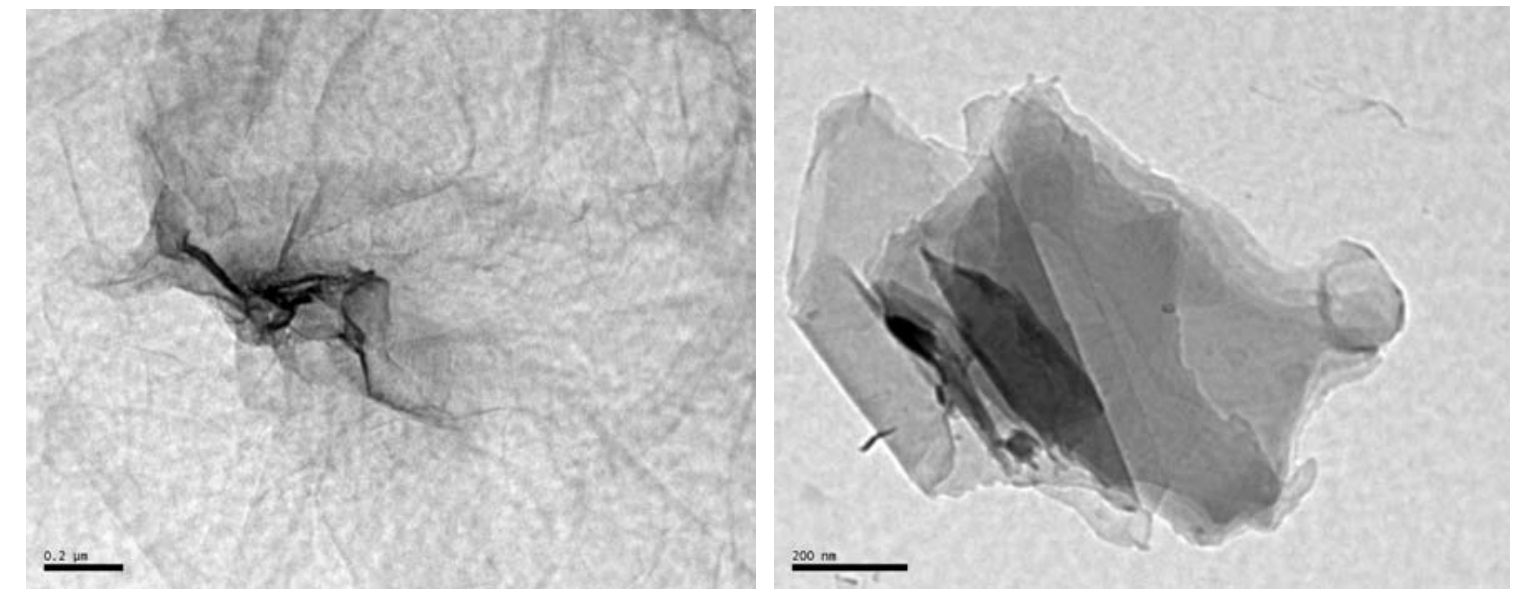

Figure 4. TEM images of GO-H-5h (left) and GO-B-5h (right). 


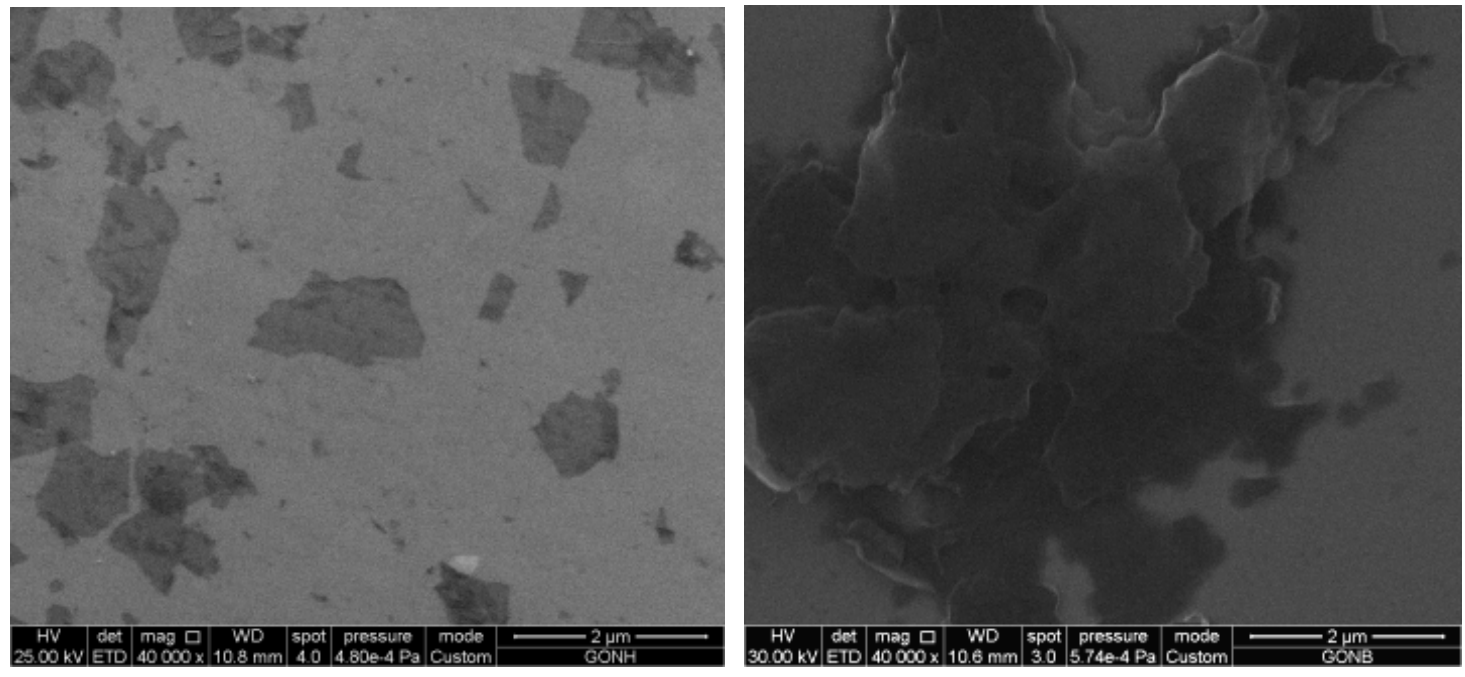

Figure 5. SEM images of GO-H-5h (left) and GO-B-5h (right). 

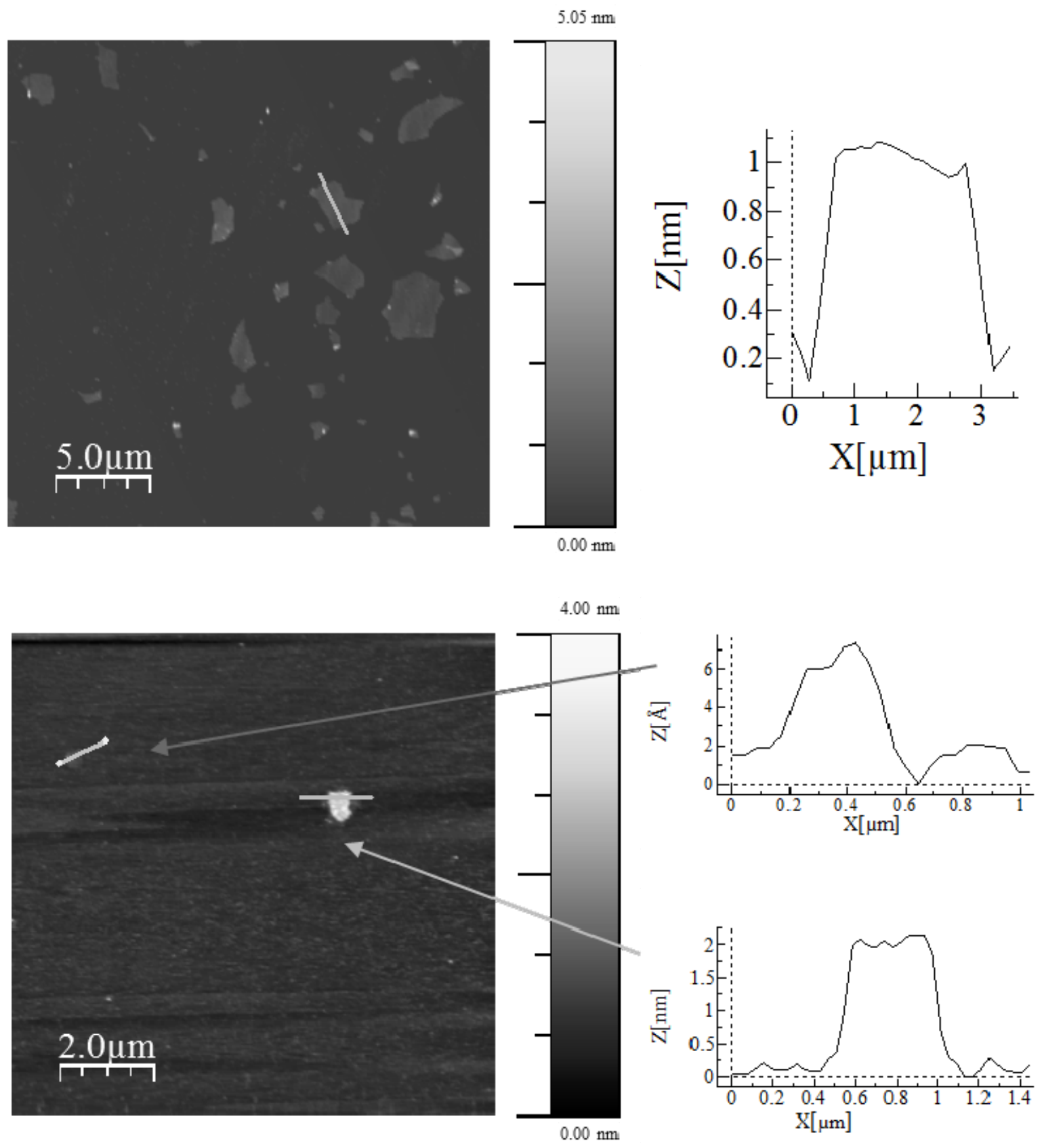

Figure 6. AFM images of GO-H-2h (top) and GO-B-5h (bottom). The blue lines indicate the sections corresponding to the traces shown on the right. 
TPD experiments were performed in both GOs (see S.I.). These allow the exfoliation temperature to be determined (when blasting occurs) and, at the same time, provide information on the type of oxygen functional groups lost with the increase in temperature. During the heating process, the oxygen functional groups of GO decompose and produce gases that build up pressure between adjacent graphene sheets, as a result of the abrupt elimination of intercalated water and oxygen groups, in the form of $\mathrm{CO}, \mathrm{CO}_{2}$. Thermal exfoliation occurs when the pressure exceeds the Van der Waals interlayer attractions [16], a higher pressure being required for shorter interlaminar distances. GO-H thermally exfoliates at lower temperature than GO-B, $150{ }^{\circ} \mathrm{C}$ and $200{ }^{\circ} \mathrm{C}$, respectively. The reason for this is to be found in a higher amount of labile groups in GO-H.

The structural differences between GO-H and GO-B are evident from the results obtained by thermogravimetric analysis (Figure 7). The TGA/DTG curves of GOs typically show the release of a small amount of water at the initial heating stage, followed by a dramatic loss at $150-300{ }^{\circ} \mathrm{C}$, corresponding to the decomposition of oxygen functional groups $[16,13]$. The products of this decomposition are mainly $\mathrm{H}_{2} \mathrm{O}$ and $\mathrm{CO}_{2}$. There is a continuous and smooth weight loss in the temperature range of $350-1000{ }^{\circ} \mathrm{C}$ (which corresponds to the loss of $\mathrm{CO}$ and $\mathrm{H}_{2}$ as corroborated by the TPD results). GO-H starts to lose weight below $150{ }^{\circ} \mathrm{C}$, maximum weight loss occurring at $200{ }^{\circ} \mathrm{C}$ (corresponding to a weight loss of about $40 \%$ ). Weight loss then progressively continues reaching $54 \%$ at $800{ }^{\circ} \mathrm{C}$. GO-B, however, does not start to lose weight until $200{ }^{\circ} \mathrm{C}$, maximum weight loss occurring at $250{ }^{\circ} \mathrm{C}$ (weight loss at $200-320^{\circ} \mathrm{C}$ was of the order $27 \mathrm{wt} \%$ ). Weight loss then continues gradually up to $900{ }^{\circ} \mathrm{C}$, where it experiences a second maximum of about 20 wt $\%$ between 900 and $1000{ }^{\circ} \mathrm{C}$. This suggests that, apart from the lower amount of 
oxygen functional groups present in GO-B (one third of the amount in GO-H, according to the elemental analysis), these groups are more stable.

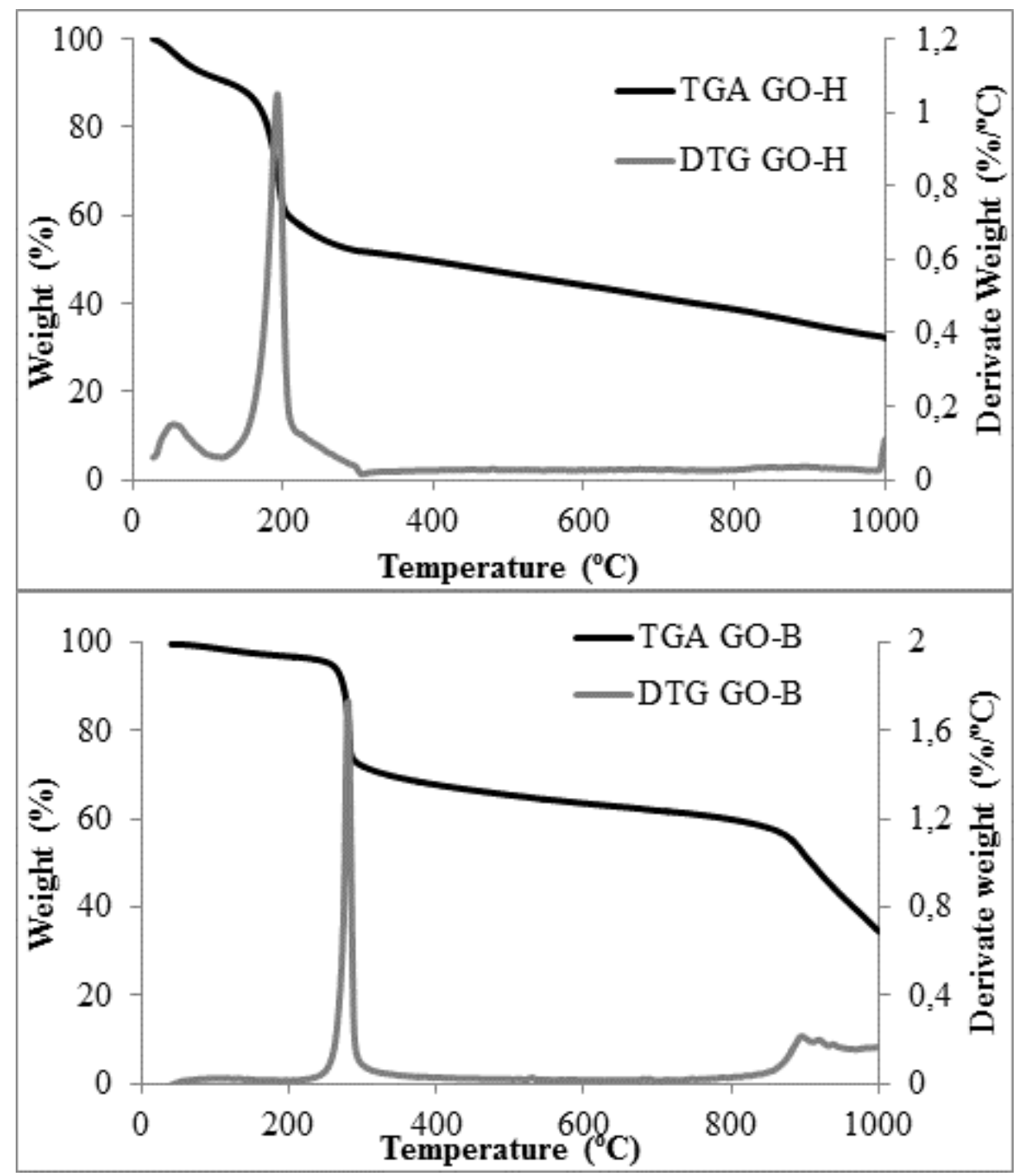

Figure 7. Thermogravimetric analysis profiles of GO-H (top) and GO-B (bottom).

The differences in the structure and thermal behavior of the two GOs affect the characteristics of graphenes resulting from their thermal exfoliation/reduction (TRGs). The type and amount of 
functional groups of TRGs were determined by XPS (Table 1). Although the oxidation was more effective with the modified Hummers method (C/O of 1.8 in GO-H vs 2.9 in GO-B), TRGH1000 and TRGH-2000 exhibit C/O ratios (of 57.8 and 332.3, respectively) which are higher than those of TRGB-1000 and TRGB-2000 (25.3 and 37.5, respectively). This confirms the previous observation by TGA. DTG evidences a more extensive reduction of oxygen functional groups in GO-H. Furthermore, only GO-B undergoes a second maximum weight loss at temperatures above $900{ }^{\circ} \mathrm{C}$. Since there is no substantial increase in the $\mathrm{C} / \mathrm{O}$ ratio of this sample in the 700 $1000{ }^{\circ} \mathrm{C}$ interval, we can conclude that the weight loss is not only caused by the elimination of oxygen containing functional groups but also by the rearrangement of its $\mathrm{C}-\mathrm{H}$ structure. It is worth mentioning that the evolution of the different type of oxygen functional groups with temperature is rather different in both samples. Although GO-H has a higher amount of all types of functional groups, their elimination at $700{ }^{\circ} \mathrm{C}$ is more pronounced than in GO-B. TRGH-1000 and TRGH-2000 contain only, $3.6 \%$ and $1.5 \%$ of hydroxyl groups, respectively. Meanwhile, there are significantly higher amounts of residual hydroxyl groups inTRGB-1000 and TRGB2000 ( 8.6 and $5.3 \%$ respectively). The most interesting finding in this study is that the restoration of the $\mathrm{sp}^{2}$-bonded $\mathrm{C}$ atoms is greater in the samples obtained by the Hummers method than by the Brodie's method, reaching $88.9 \%$ in TRGH-2000 while in the case of TRGB-2000 it reaches $81.8 \%$. This is surprising as the Brodie's method is less aggressive and the resulting GO is less functionalized. This means that the higher thermal stability of the oxygen functional groups introduced by the Brodie's method makes their removal more difficult and that it generates more defects. 
Considering the theoretical studies reported by Bagri et al [15], it can be proposed that GO-B contains conjugated epoxy groups and hydroxyl, which, at moderate temperatures lead to the incorporation of oxygen in-plane as ether groups or out-of-plane as carbonyl groups. These groups are highly stable and thus the $\mathrm{Csp}^{2}$ structure of the carbon lattice is not fully recovered. In contrast, the presence of less conjugated oxygen functional groups in GO-H facilitates their thermal removal, and no oxygen is incorporated into the carbon lattice, resulting in a better restoration of the $\mathrm{sp}^{2}$ structure. This is consistent with the higher oxygen content remaining in the GO-B sample even after treatment at $2000^{\circ} \mathrm{C}(2.6 \%)$ and with the higher $\mathrm{I}_{\mathrm{D}} / \mathrm{I}_{\mathrm{G}}$ ratio in TRGB$2000(0.33)$ than in TRGH-2000 (0.09). 
Table 1. Main characteristics of samples.

\begin{tabular}{|c|c|c|c|c|c|c|c|c|c|c|c|c|c|c|c|c|c|}
\hline & \multicolumn{5}{|c|}{ Elemental Analysis (wt. \%) } & \multicolumn{8}{|c|}{ XPS } & \multirow{2}{*}{$\frac{\mathrm{S}_{\mathrm{BET}}^{\mathrm{a}}}{\left(\mathrm{m}^{2} \mathrm{~g}^{-1}\right)}$} & \multicolumn{3}{|c|}{ Raman } \\
\hline & $\mathrm{C}$ & $\mathrm{H}$ & $\mathrm{O}$ & $\mathrm{N}$ & $S$ & $\mathrm{C} / \mathrm{O}$ & $\begin{array}{c}\mathrm{O} \\
(\%)\end{array}$ & $\begin{array}{c}\mathrm{C} \\
(\%)\end{array}$ & $\begin{array}{c}\operatorname{Csp}^{2} \\
(\%)\end{array}$ & $\begin{array}{c}\operatorname{Csp}^{3} \\
(\%)\end{array}$ & $\begin{array}{c}\mathrm{C}-\mathrm{OH} \\
(\%)\end{array}$ & $\begin{array}{c}\mathrm{C}-\mathrm{O}-\mathrm{C} \\
(\%)\end{array}$ & $\begin{array}{c}\mathrm{C}(\mathrm{O}) \mathrm{OH} \\
(\%)\end{array}$ & & $\mathrm{I}_{\mathrm{D}} / \mathrm{I}_{\mathrm{G}}^{\mathrm{b}}$ & $\begin{array}{c}\mathrm{W}_{\mathrm{D}}^{\mathrm{c}} \\
\left(\mathrm{cm}^{-1}\right)\end{array}$ & $\begin{array}{c}W_{G}^{d} \\
\left(\mathrm{~cm}^{-1}\right)\end{array}$ \\
\hline GO-H & 48.0 & 2.2 & 47.8 & 0.0 & 2.0 & 1.8 & 35.2 & 64.8 & 32.2 & 12.8 & 36.2 & 14.3 & 4.4 & --- & 0.88 & 1348 & 1592 \\
\hline TRGH-700 & 87.8 & 0.8 & 11.1 & 0.0 & 0.3 & 9.2 & 9.8 & 90.2 & 74.6 & 15.1 & 8.5 & 0.6 & 1.2 & 390 & 0.91 & 1354 & 1592 \\
\hline TRGH-1000 & 97.9 & 0.1 & 1.1 & 0.0 & 0.9 & 57.8 & 1.7 & 98.3 & 82.4 & 13.9 & 3.6 & 0.0 & 0.0 & 300 & 1.28 & 1335 & 1570 \\
\hline TRGH-2000 & 99.5 & 0.0 & 0.5 & 0.0 & 0.0 & 332.3 & 0.3 & 99.7 & 88.9 & 9.6 & 1.5 & 0.0 & 0.0 & 140 & 0.09 & 1350 & 1580 \\
\hline GO-B & 70.1 & 0.9 & 28.2 & 0.0 & 0.0 & 2.9 & 25.7 & 74.3 & 39.2 & 14.5 & 32.4 & 11.2 & 2.7 & --- & 0.88 & 1332 & 1565 \\
\hline TRGB-700 & 90.1 & 0.2 & 9.7 & 0.0 & 0.0 & 13.1 & 7.1 & 92.9 & 75.0 & 13.1 & 9.2 & 1.8 & 0.9 & 660 & 0.86 & 1353 & 1578 \\
\hline TRGB-1000 & 98.0 & 0.3 & 1.6 & 0.1 & 0.0 & 25.3 & 3.8 & 96.2 & 77.3 & 14.1 & 8.6 & 0.0 & 0.0 & 570 & 1.10 & 1335 & 1570 \\
\hline TRGB-2000 & 99.3 & 0.0 & 0.7 & 0.0 & 0.0 & 37.5 & 2.6 & 97.4 & 81.8 & 13.0 & 5.3 & 0.0 & 0.0 & 140 & 0.33 & 1364 & 1588 \\
\hline
\end{tabular}

\footnotetext{
, BET surface area.

, ratio of intensities of band D and G in the Raman spectra

c, position of band D in the Raman spectra

$\mathrm{d}$, position of band $\mathrm{G}$ in the Raman spectra
} 
While no significant differences were observed by TEM in the two series of TRGs (see S.I.), SEM (Figure 8) of the as-prepared powder samples shows important differences between those obtained at 700 and $1000{ }^{\circ} \mathrm{C}$ by the two methods. TRGH-700 and TRGH-1000 show the typical randomly oriented graphene sheets previously reported in other studies [17, 18, 23] (Figure 8), while TRGB-700 and TRGB-1000 exhibit some areas with "accordion-type" sheets typical of expanded graphite [27] which indicates the partial exfoliation of the GO-B (as in the case of the ultrasounds exfoliation discussed above). This may be the factor responsible for their large BET surface area (660 and $570 \mathrm{~m}^{2} \mathrm{~g}^{-1}$; vs 390 and $300 \mathrm{~m}^{2} \mathrm{~g}^{-1}$ in TRGH-700 and TRGH-1000. TRGH2000 and TRGB-2000, however, both exhibit the typical shape of randomly oriented graphene sheets. This means that the gases produced by the removal of the more stable functional groups above $1000{ }^{\circ} \mathrm{C}$, as observed by DTG and TPD (see S.I.) completed the exfoliation of GO-B. 


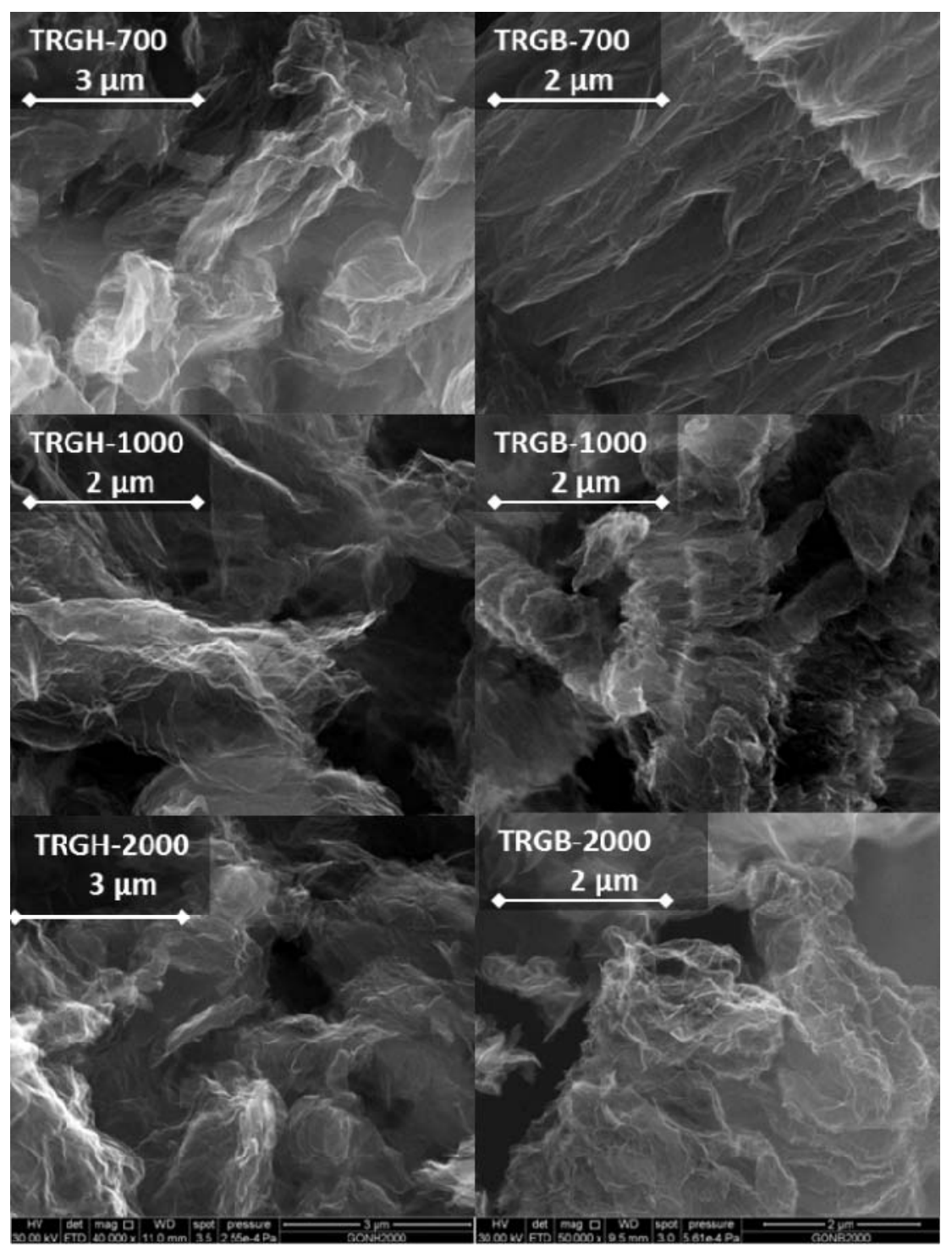

Figure 8. SEM images of powder TRGs obtained at $700{ }^{\circ} \mathrm{C}, 1000{ }^{\circ} \mathrm{C}$ and $2000{ }^{\circ} \mathrm{C}$.

\section{CONCLUSIONS}

"Tailor made" graphene sheets of different size and surface area, with an accurate structure and functionality, can be obtained by controlling the oxidation process of the graphite and the thermal reduction of the oxide. The Brodie's method introduces a smaller amount of oxygen than Hummers, but favors the formation of conjugated epoxy groups and hydroxyl, which, at 
moderate temperatures leads to the incorporation of oxygen in-plane as ether groups or out-ofplane as carbonyl groups. These groups are highly stable. Thus the $\mathrm{Csp}^{2}$ structure of the carbon lattice is not fully recovered and residual oxygen remains even after treatment at $2000^{\circ} \mathrm{C}$. In contrast, the presence of less conjugated oxygen groups in GO-H facilitates their thermal removal, and no oxygen is incorporated into the carbon lattice, resulting in a better restoration of the $\mathrm{sp}^{2}$ structure. In short, a larger restoration of the pristine graphite 2D structure is achieved by Hummers oxidation method.

\section{ACKNOWLEDGMENTS}

The authors thank MICINN and European Union (CONSOLIDER INGENIO 2010, Ref. CSD2009-00050, MAT2010-16194) for their financial support. Dr. Patricia Alvarez thanks MICINN for her Ramon y Cajal contract. 


\section{REFERENCES}

[1] Novoselov KS, Fal'ko VI, Colombo L, Gellert PR, Schwab MG, Kim K. A road map for graphene. Nature 2012; 490: 192-200.

[2] Dreyer DR, Park S, Bielawski CW, Ruoff RS. The chemistry of graphene oxide. Chem Soc Rev 2010; 39: 228-40.

[3] Kim H, Abdala AA, Macosko CW. Graphene/Polymer Nanocomposites. Macromolecules 2010; 43: 6515-30.

[4] Verdejo R, Bernal MM, Romasanta LJ, Lopez-Manchado MA. Graphene filled polymer nanocomposites. Journal of Materials Chemistry 2011; 21: 3301-10.

[5] Singh V, Joung D, Zhai L, Das S, Khondaker SI, Seal S. Graphene based materials: Past, present and future. Progress in Materials Science 2011; 56: 1178-271.

[6] Botas C, Álvarez P, Blanco C, Santamaría R, Granda M, Ares P, et al. R. The effect of the parent graphite on the structure of graphene oxide. Carbon 2012; 50: 275-82.

[7] Poh HL, Sanék F, Ambrosi A, Zhao G, Soferb Z, Pumera M. High-pressure hydrogenation of graphene: Towards graphane. Nanoscale 2012; 4: 3515-22.

[8] Marcano DC, Kosynkin DV, Berlin JM, Sinitskii A, Sun Z, Slesarev A, et al. Improved synthesis of graphene oxide. ACS Nano 2010; 4: 4806-14.

[9] Gao X, Jang J, Nagase S. Hydrazine and thermal reduction of graphene oxide: Reaction mechanisms, product structures, and reaction design. J Phys Chem C 2010; 114: 832-42.

[10] Pei S, Cheng H. The reduction of graphene oxide. Carbon 2011; 50: 3210-28.

[11] Lerf A, He H, Forster M, Klinowski J. Structure of Graphite Oxide Revisited. J. Phys. Chem. B 1998; 102: 4477-82. 
[12] Szabo T, Berkesi O, Forgo P, Josepovits K, Sanakis Y, Petridis D and Dekany I. Evolution of Surface Functional Groups in a Series of Progressively Oxidized Graphite Oxides. Chem. Mater. 2006; 18: 2740-9.

[13] Dreyer DR, Park S, Bielawski CW, Ruoff RS. The chemistry of graphene oxide Chem. Soc. Rev. 2010; 39: 228-40.

[14] Botas C, Álvarez P, Blanco C, Gutiérrez MD, Ares P, Zamani R, et al. Tailored graphene materials by chemical reduction of graphene oxides of different atomic structure. RSC Adv 2012; 2: 9643-50.

[15] Bagri A, Mattevi C, Acik M, Chabal Y, Chhowalla M, Shenoy V. Structural evolution during the reduction of chemically derived graphene oxide. Nat Chem 2010; 2: 581-7.

[16] McAllister MJ, Li J, Adamson DH, Schniepp HC, Abdala AA, Liu J, et al. Single sheet functionalized graphene by oxidation and thermal expansion of graphite. Chem Mater 2007; 19: 4397-404.

[17] Wang G, Shen X, Yao Y, Park J. Graphene nanosheets for enhanced lithium storage in lithium ion batteries. Carbon 2009; 47: 2049-53.

[18] González Z, Botas C, Álvarez P, Roldán S, Blanco C, Santamaría R, et al. Thermally reduced graphite oxide as positive electrode in Vanadium Redox Flow Batteries. Carbon 2012; 50: $828-34$.

[19] Wajid AS, Das S, Irin F, Ahmed HST, Shelburne JL, Parviz D, et al. Polymer-stabilized graphene dispersions at high concentrations in organic solvents for composite production. Carbon 2012; 50: 526-34.

[20] Hummers WS, Offeman RE. Preparation of graphitic oxide. J Am Chem Soc 1958; 80: $1339-40$. 
[21] Brodie BC. Hydration behavior and dynamics of water molecules in graphite oxide. Ann Chim Phys 1860; 59: 466-72.

[22] You S, Luzana SM, Szabó T, Talyzin AV. Effect of synthesis method on solvation and exfoliation of graphite oxide. Carbon 2013; 52: 171-80.

[23] Botas C, Álvarez P, Blanco C, Santamaría R, Granda M, Gutiérrez MD, et al. Critical temperatures in the synthesis of graphene-like materials by thermal exfoliation-reduction of graphite oxide. Carbon 2013; 52: 476-85.

[24] Yang D, Velamakanni A, Bozoklu G, Park S, Stoller M, Piner RD, et al. Chemical analysis of graphene oxide films after heat and chemical treatments by $\mathrm{x}$-ray photoelectron and microraman spectroscopy. Carbon 2009; 47: 145-52.

[25] Ferrari AC. Raman spectroscopy of graphene and graphite: Disorder, electron-phonon coupling, doping and nonadiabatic efffects. Solid State Communications 2007; 143: 47-57

[26] Casiraghi C, Hartschuh A, Qian H, Piscanec S, Georgi C, Fasoli A, et al. Raman spectroscopy of graphene edges. Nano Letters 2009; 9: 1433-41.

[27] Potts JR, Shankar O, Murali S, Dub L, Ruoff RS. Latex and two-roll mill processing of thermally-exfoliated graphite oxide/natural rubber nanocomposites. Compos Sci Technol 2013; 74: $166-72$. 


\section{Figure captions}

Figure 1. XRD spectra of parent graphite and GOs.

Figure 2. Images of GO-H (left) and GO-B (right) in water (without ultrasonication).

Figure 3. UV-Vis spectra of GrO-H (left) and GrO-B (right) at different sonication times (1, 5 and $15 \mathrm{~h})$.

Figure 4. TEM images of GO-H-5h (left) and GO-B-5h (right).

Figure 5. SEM images of GO-H-5h (left) and GO-B-5h (right).

Figure 6. AFM images of GO-H-2h (top) and GO-B-5h (bottom). The blue lines indicate the sections corresponding to the traces shown on the right

Figure 7. Thermogravimetric analysis profiles of GO-H (top) and GO-B (botton).

Figure 8. SEM images of powder TRGs obtained at $700{ }^{\circ} \mathrm{C}, 1000^{\circ} \mathrm{C}$ and $2000{ }^{\circ} \mathrm{C}$. 
Table captions

Table 1. Main characteristics of samples. 